Suportting Information

ACS Sustainable Chemistry \& Engineering

\title{
Ionic liquids can enable the recycling of fluorinated greenhouse gases
}

Luiz Fernando Lepre,[a] Dune Andre,[b] Sandrine Denis-Quanquin,[a] Arnaud Gautier, [b] Agilio Padua, ${ }_{[a]}$ and Margarida Costa Gomes ${ }^{*}{ }_{[a]}$

Number of pages : 9

Number of figures : 4

Number of tables : 5

[a] Laboratoire de Chimie, Ecole Normale Supérieure de Lyon \& CNRS, 69364 Lyon, France.

[b] Institut de Chimie de Clermont-Ferrand, Université Clermont Auvergne, 24 av Blaise Pascal, 63178 Aubière, France

*Corresponding authors: margarida.costa-gomes@ens-lyon.fr; agilio.padua@ens-lyon.fr 
Table S1. Experimental values of $R 134$ a solubility in the $\left[\mathrm{C}_{2} \mathrm{C}_{1} \mathrm{Im}\right][\mathrm{NTf} 2]$ ionic liquid expressed as the R134a mole fraction (XR134a) and the experimental equilibrium pressure (p).

\begin{tabular}{ccc}
\hline $\mathrm{p} /$ bar & XR134a \\
\hline & $303 \mathrm{~K}$ & \\
0.24864 & & 0 \\
0.49899 & 0.03082 \\
0.99831 & 0.06037 \\
1.99944 & 0.11688 \\
2.99517 & 0.22381 \\
3.49947 & 0.32311 \\
\hline
\end{tabular}

Table S2. Experimental values of R134a solubility in the [ $\left.\mathrm{C}_{8} \mathrm{C}_{1} \mathrm{Im}\right]\left[\mathrm{NTf} f_{2}\right]$ ionic liquid expressed as the R134a mole fraction (XR134a) and the experimental equilibrium pressure $(p)$.

\begin{tabular}{ccc}
\hline $\mathrm{p} / \mathrm{bar}$ & $\mathrm{XR} 134 \mathrm{a}$ & $\mathrm{deV}$ \\
\hline & $303 \mathrm{~K}$ & 0 \\
0.24892 & 0 & 0.00175 \\
0.49823 & 0.03535 & 0.00201 \\
0.99492 & 0.06829 & 0.00149 \\
1.99819 & 0.13059 & 0.00205 \\
2.9951 & 0.24506 & 0.00138 \\
3.49268 & 0.34885 & 0 \\
\hline 0 & 0.39756 & 0 \\
0.24933 & $323 \mathrm{~K}$ & 0.00136 \\
0.49899 & 0 & 0.00127 \\
0.99893 & 0.02349 & 0.00094 \\
1.99501 & 0.04513 & 0.00127 \\
2.99876 & 0.0873 & 0.00053 \\
3.49628 & 0.16517 & 0 \\
\hline & 0.23666 & 0 \\
0.24837 & 0.2697 & 0.00097 \\
0.49941 & $343 \mathrm{~K}$ & 0.00111 \\
0.99831 & 0 & 0.00070 \\
1.9966 & 0.01607 & 0.00045 \\
2.99752 & 0.0313 & 0.00019 \\
3.48867 & 0.06153 & 0 \\
\hline
\end{tabular}


Table S3. Experimental values of R134a solubility in the $\left[\mathrm{C}_{8} \mathrm{H}_{4} \mathrm{~F}_{13} \mathrm{C}_{1} \operatorname{lm}\right][\mathrm{NTf} 2]$ ionic liquid expressed as the R134a mole fraction (XR134a) and the experimental equilibrium pressure $(p)$.

\begin{tabular}{ccc}
\hline $\mathrm{p} /$ bar & XR134a & dev \\
\hline & $303 \mathrm{~K}$ & 0 \\
0.24809 & 0 & 0.00467 \\
0.49982 & 0.04465 & 0.00467 \\
0.99533 & 0.08407 & 0.00579 \\
1.9885 & 0.15849 & 0.00254 \\
2.99191 & 0.28974 & 0.00163 \\
3.4931 & 0.40547 & 0 \\
\hline 0 & 0.4586 & 0 \\
0.24837 & $323 \mathrm{~K}$ & 0.00282 \\
0.49865 & 0 & 0.00309 \\
0.99554 & 0.03028 & 0.00243 \\
1.99916 & 0.05695 & 0.00195 \\
2.99344 & 0.10868 & 0.00053 \\
3.49268 & 0.20199 & 0 \\
\hline & 0.28509 & \\
\hline 0 & 0.32301 & 0 \\
0.44885 & $343 \mathrm{~K}$ & 0.00236 \\
0.9972 & 0 & 0.00237 \\
1.99113 & 0.02095 & 0.00136 \\
2.99067 & 0.04193 & 0.00110 \\
3.48936 & 0.08068 & 0.00076 \\
\hline
\end{tabular}


Table S4. Experimental values of $\mathrm{R} 134 \mathrm{a}$ solubility in the $\left[\mathrm{C}_{8} \mathrm{C}_{1} \mathrm{Im}\right][\mathrm{BETI}]$ ionic liquid expressed as the R134a mole fraction (XR134a) and the experimental equilibrium pressure $(p)$.

\begin{tabular}{ccc}
\hline $\mathrm{p} /$ bar & XR134a & dev \\
\hline 0 & $303 \mathrm{~K}$ & 0 \\
0.24871 & 0 & 0.00212 \\
0.49892 & 0.04243 & 0.00233 \\
0.99769 & 0.08204 & 0.00237 \\
1.99238 & 0.15507 & 0.00114 \\
2.99434 & 0.28576 & 0.00092 \\
3.48853 & 0.39941 & 0 \\
\hline 0 & 0.45229 & 0 \\
0.24857 & $323 \mathrm{~K}$ & 0.00236 \\
0.49913 & 0 & 0.00166 \\
0.99859 & 0.02808 & 0.00188 \\
1.99736 & 0.05398 & 0.00081 \\
2.99371 & 0.10309 & 0.00086 \\
3.49476 & 0.19293 & 0 \\
\hline & 0.27289 & 0 \\
0.24837 & 0.30984 & 0.00125 \\
0.49913 & $343 \mathrm{~K}$ & 0.00130 \\
0.9972 & 0 & 0.00062 \\
1.99259 & 0.01906 & 0.00032 \\
2.98714 & 0.03736 & 0.00006 \\
3.49047 & 0.07356 & 0 \\
\hline
\end{tabular}


Table S5. Experimental values of $\mathrm{R} 134 \mathrm{a}$ solubility in the $\left[\mathrm{C}_{8} \mathrm{H}_{4} \mathrm{~F}_{13} \mathrm{C}_{1} / \mathrm{m}\right][\mathrm{BETI}]$ ionic liquid expressed as the R134a mole fraction (XR134a) and the experimental equilibrium pressure $(p)$.

\begin{tabular}{ccc}
\hline $\mathrm{p} / \mathrm{bar}$ & $\mathrm{XR} 134 \mathrm{a}$ & $\mathrm{dev}$ \\
\hline & $303 \mathrm{~K}$ & 0 \\
0.24823 & 0 & 0.00963 \\
0.49885 & 0.04052 & 0.01202 \\
0.99873 & 0.08224 & 0.01099 \\
1.99992 & 0.16033 & 0.00306 \\
2.99911 & 0.30117 & 0.00077 \\
3.49601 & 0.41938 & 0 \\
\hline 0 & 0.47614 & 0 \\
0.24892 & $323 \mathrm{~K}$ & 0.00242 \\
0.49934 & 0 & 0.00224 \\
0.99935 & 0.02084 & 0.00173 \\
1.99951 & 0.05043 & 0.00156 \\
2.99648 & 0.10631 & 0.00103 \\
3.49116 & 0.20583 & 0 \\
\hline & 0.29278 & 0 \\
0.24864 & 0.33202 & 0.00173 \\
0.49948 & $343 \mathrm{~K}$ & 0.00193 \\
0.99886 & 0 & 0.00077 \\
1.99529 & 0.02158 & 0.00060 \\
2.99281 & 0.04324 & 0.00002 \\
3.4985 & 0.08424 & 0 \\
\hline
\end{tabular}



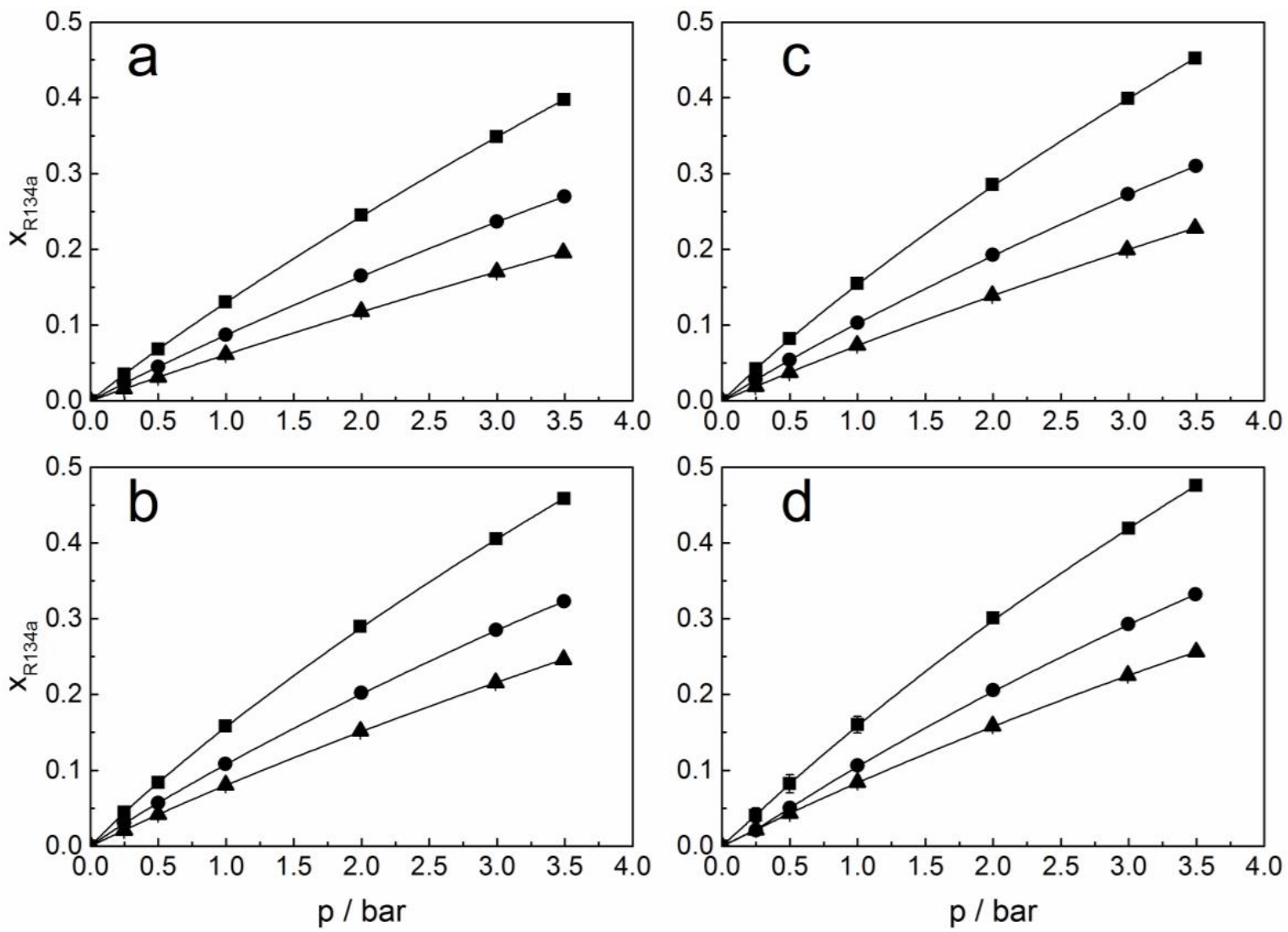

Figure S1. R134a mole fraction solubility in (a) $\left[\mathrm{C}_{8} \mathrm{C}_{1} \mid \mathrm{m}\right]\left[\mathrm{NTf} f_{2}\right]$, (b)

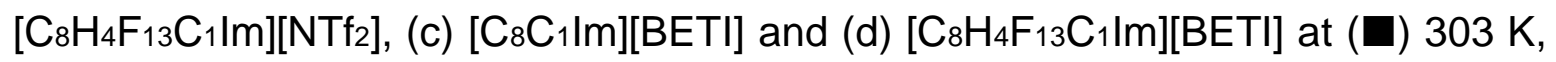
(-) $323 \mathrm{~K}$ and $(\mathbf{\Delta}) 343 \mathrm{~K}$ as a function of pressure. Solid lines are just eye guides. 

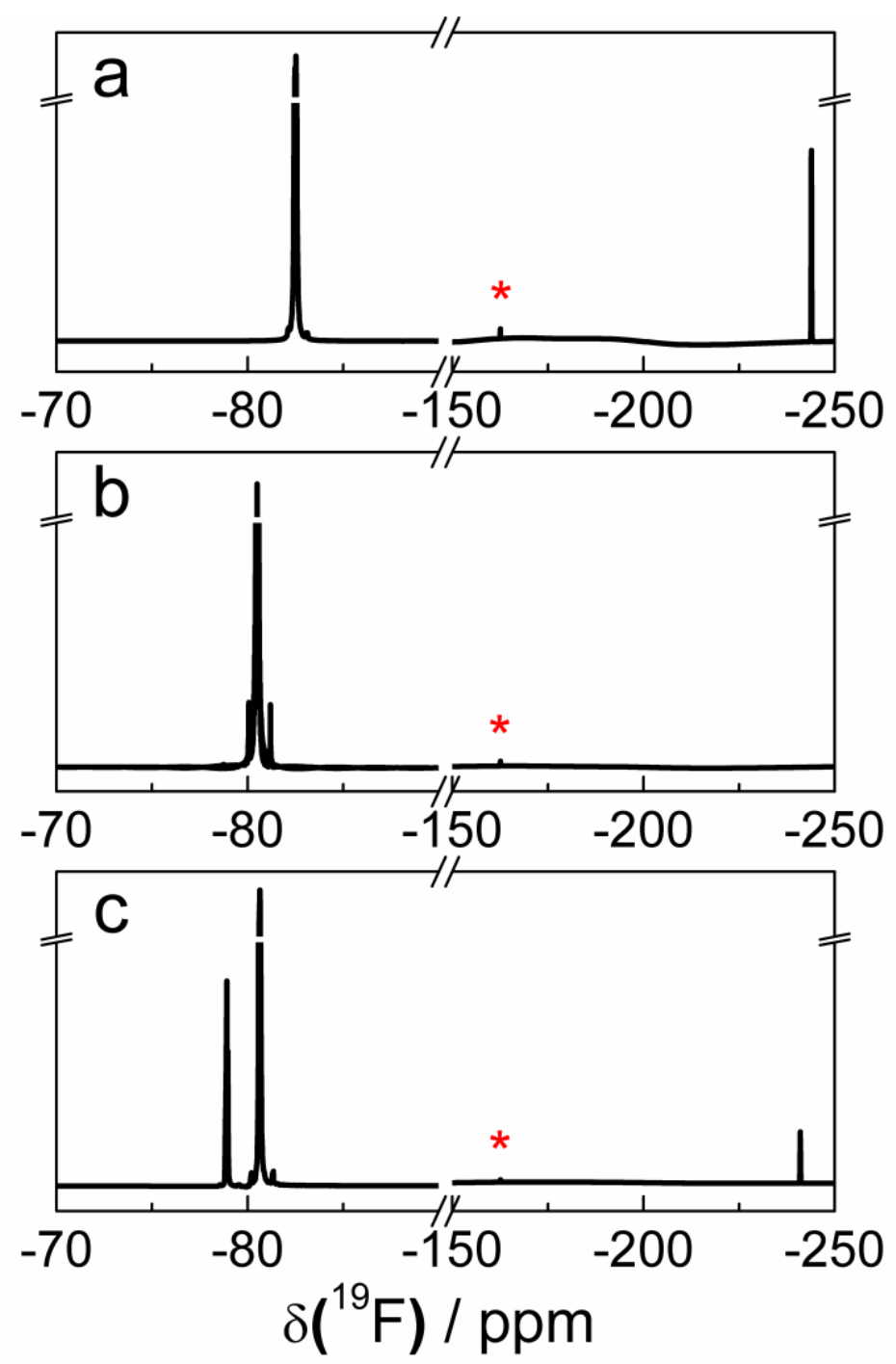

Figure S2. ${ }_{19} \mathrm{~F} \mathrm{NMR}$ spectra at $298 \mathrm{~K}$ of (a) pure $\mathrm{R} 134 \mathrm{a}$ at 5 bar, (b) pure $\left[\mathrm{C}_{8} \mathrm{C}_{1} \mathrm{Im}\right]\left[\mathrm{NTf}_{2}\right]$ under vacuum and (c) solution of $\mathrm{R} 134 \mathrm{a}$ in $\left[\mathrm{C}_{8} \mathrm{C}_{1} \mathrm{Im}\right]\left[\mathrm{NTf} f_{2}\right]$ at 5 bar. The symbol * stands for the hexafluorobenzene $\left(\mathrm{C}_{6} \mathrm{~F}_{6}\right)$ signal at $\delta\left({ }_{19} \mathrm{~F}\right)=-162.6 \mathrm{ppm}$ used as a reference. The $\mathrm{C}_{6} \mathrm{~F}_{6} 6$ solution in DMSO-d 6 was located in a closed capillary inside the NMR tube so that any contact between the reference and sample could be prevented. 

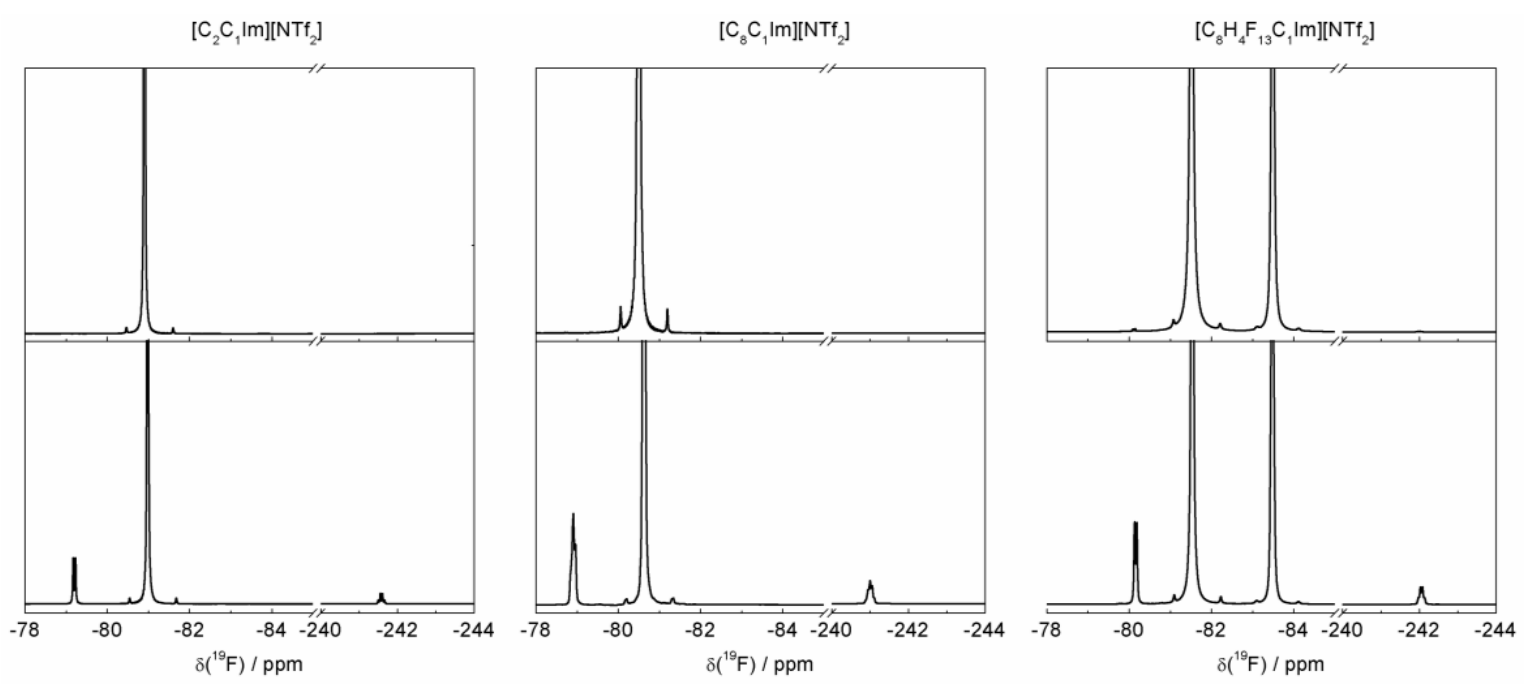

Figure S3. ${ }_{19} \mathrm{~F}$ NMR spectra at $298 \mathrm{~K}$ of $\left[\mathrm{C}_{2} \mathrm{C}_{1} \mid \mathrm{m}\right][\mathrm{NTf} 2],\left[\mathrm{C}_{8} \mathrm{C}_{1} \mid \mathrm{m}\right]\left[\mathrm{NTf} f_{2}\right]$ and $\left[\mathrm{C}_{8} \mathrm{H}_{4} \mathrm{~F}_{13} \mathrm{C}_{1} \mathrm{Im}\right]\left[\mathrm{NTf}_{2}\right]$ under vacuum (top panel) and after reaching equilibrium with $\mathrm{R} 134 \mathrm{a}$ at 5 bar (bottom panel). Both the ${ }_{19} \mathrm{~F} \delta\left(\mathrm{CF}_{3}\right)$ and $\delta\left(\mathrm{CH}_{2} \mathrm{~F}\right)$ peaks of $\mathrm{R} 134 \mathrm{a}$ are seen in the ionic liquid solutions. 

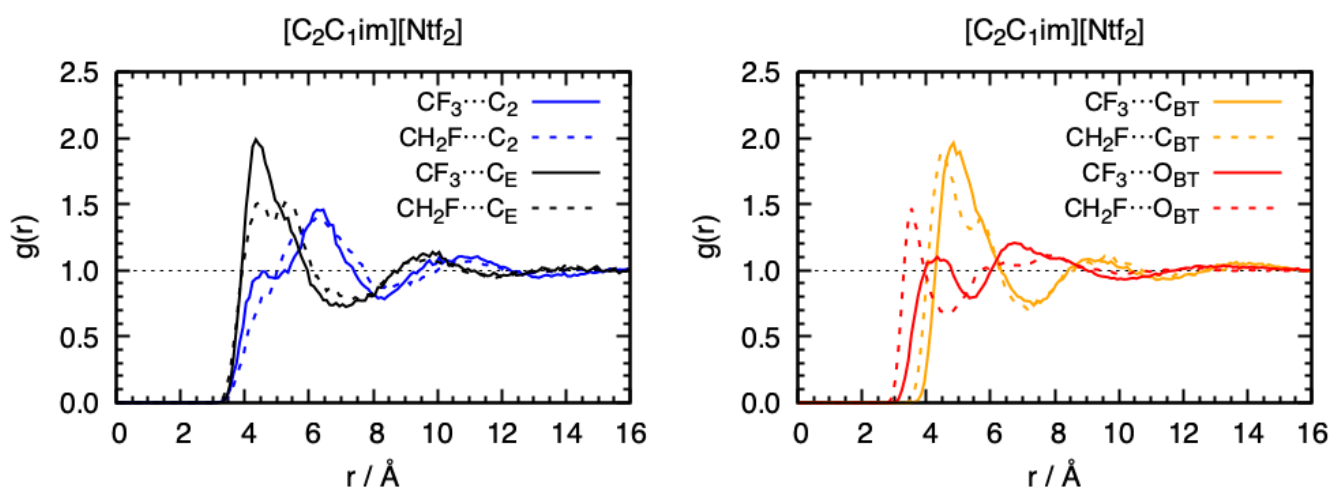

$\left[\mathrm{C}_{8} \mathrm{C}_{1}\right.$ im] $\left[\mathrm{Ntf}_{2}\right]$

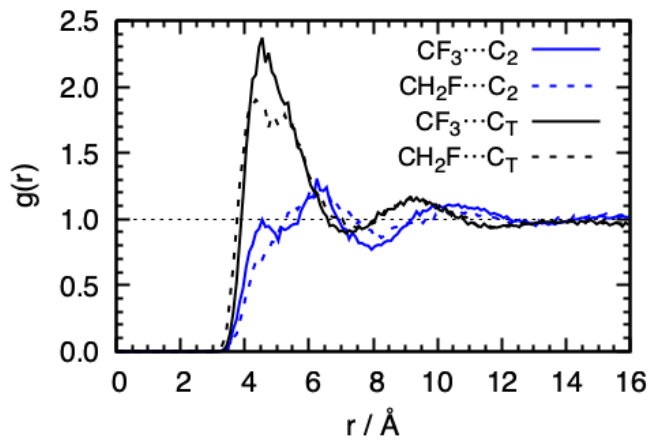

$\left[\mathrm{C}_{8} \mathrm{C}_{1} \mathrm{im}\right]\left[\mathrm{Ntf}_{2}\right]$
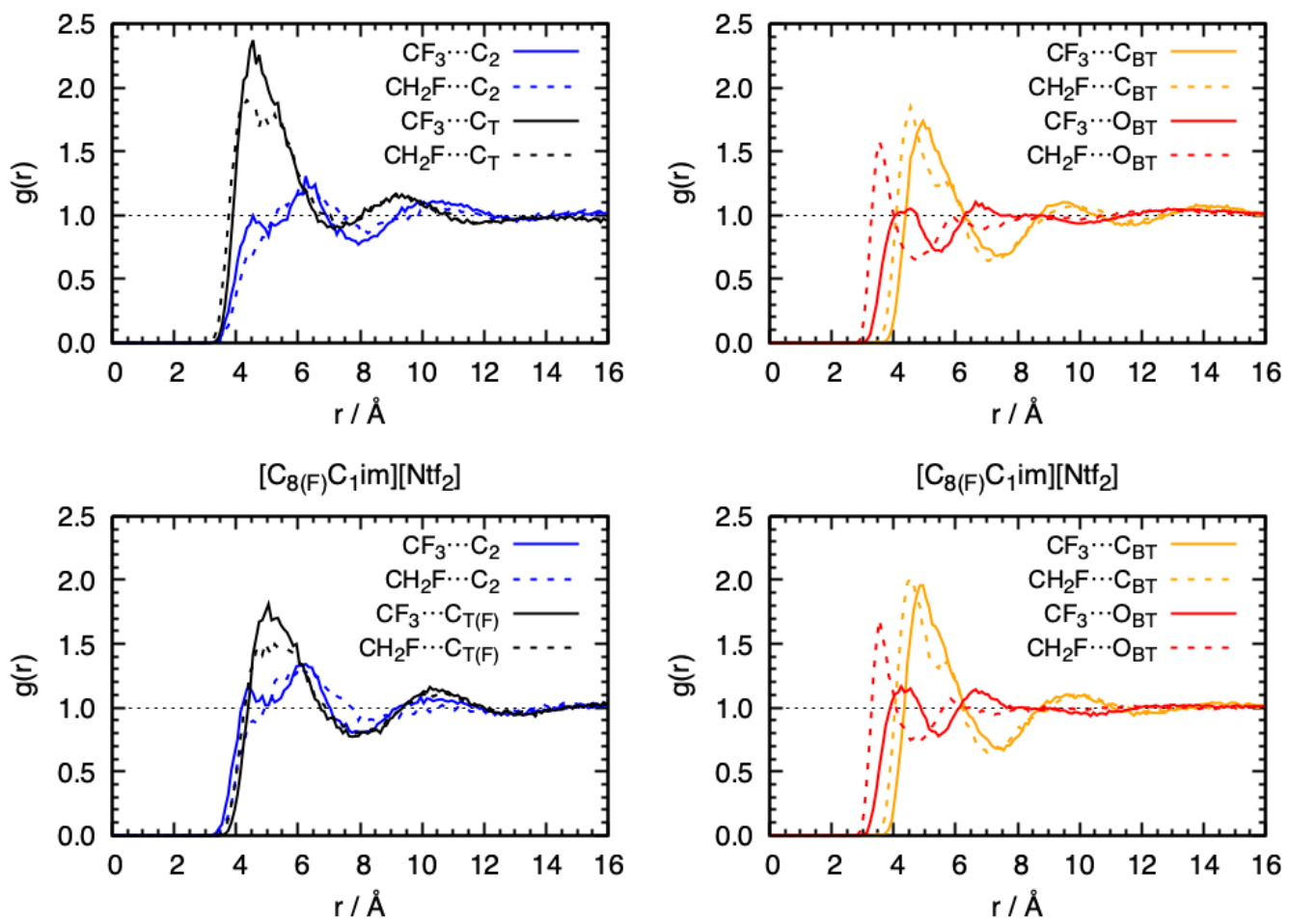

$\left[\mathrm{C}_{8} \mathrm{C}_{1} \mathrm{im}\right][\mathrm{BETl}]$

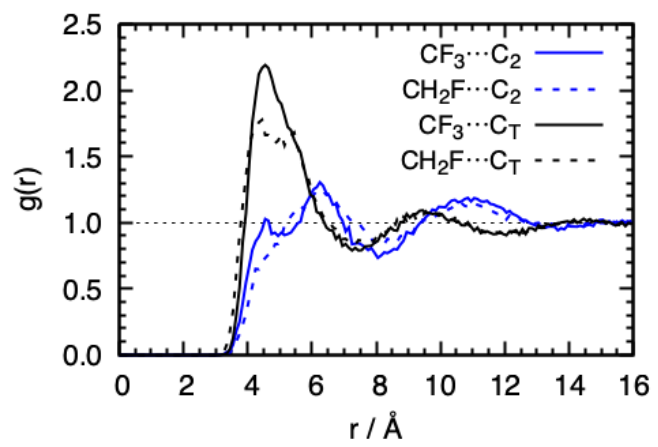

$\left[\mathrm{C}_{8} \mathrm{C}_{1} \mathrm{im}\right][\mathrm{BETI}]$

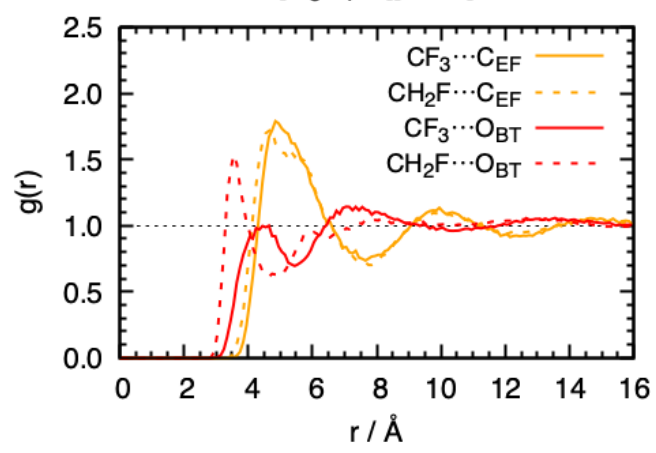

Figure S4. Radial distribution functions of atomic sites in the ionc around the R134a solute. 